\title{
Hypertrophic cardiomyopathy in young Maine Coon cats caused by the p.A31P cMyBP-C mutation - the clinical significance of having the mutation
}

\author{
Mia TN Godiksen ${ }^{1,2}$, Sara Granstrøm¹,2, Jørgen Koch², Michael Christiansen ${ }^{1 *}$
}

\begin{abstract}
Background: In Maine Coon (MC) cats the C.91G > C mutation in the gene MYBPC3, coding for cardiac myosin binding protein $\mathrm{C}$ (cMyBP-C), is associated with feline hypertrophic cardiomyopathy (fHCM). The mutation causes a substitution of an alanine for a proline at residue 31 (p.A31P) of cMyBP-C. The pattern of inheritance has been considered autosomal dominant based on a single pedigree. However, larger studies are needed to establish the significance of cats being heterozygous or homozygous for the mutation with respect to echocardiographic indices and the probability of developing $\mathrm{fHCM}$. The objective of the present study was to establish the clinical significance of being homozygous or heterozygous for the P.A31P cMyBP-C mutation in young to middle-aged cats.

Methods: The cohort consisted of 332 MC cats, 282 cats < 4 years (85\%). All cats were examined by 2-D and Mmode echocardiography. DNA was extracted from blood samples or buccal swabs and screened for the p.A31P CMyBP-C mutation in exon 3 of the gene, using polymerase chain reaction followed by DNA sequencing.

Results: The fHCM prevalence was $6.3 \%$ in the cohort. Eighteen cats were homozygous and 89 cats were heterozygous for the mutation. The odds ratio for having fHCM for homozygous cats was 21.6 (95\% confidence interval 7.01-66.2) - when the group of equivocal cats was categorized as non-affected. Overall, $50 \%$ of the cats that were homozygous for the mutation had fHCM. p.A31P heterozygosity was not associated with a significant odds ratio for $\mathrm{fHCM}$. In cats in the 4 to 6 years of age range a similar, non significant, odds ratio was seen in heterozygous cats. Only two cats over four years were homozygous and both were diagnosed with fHCM.

Conclusion: As there is no significant odds ratio associated with being heterozygous for the PA31P CMyBP-C mutation at this age, the mutation must have a very low penetrance in this group. From our data it would appear that most MC cats that develop fHCM due to the p.A31P mutation prior to the age of approximately 6 years do so because they are homozygous for this mutation.
\end{abstract}

\section{Background}

Hypertrophic cardiomyopathy (HCM), in humans, is a primary disorder of the myocardium that most commonly results from mutations in genes that encode for sarcomeric proteins. Feline HCM (fHCM) is a clinically heterogeneous disorder which is characterised by localized or generalized concentric left ventricular

\footnotetext{
* Correspondence: mic@ssi.dk

'Department of Clinical Biochemistry and Immunology, Statens Serum Institut, Artillerivej 5, DK-2300 Copenhagen, Denmark

Full list of author information is available at the end of the article
}

hypertrophy and diastolic dysfunction [1-7]. Affected cats may progress into congestive heart failure, thromboembolic events or sudden cardiac death [8]. Not much is known about the genetics underlying $\mathrm{fHCM}$ and presently only two mutations have been found $[4,5]$.

The Maine Coon (MC) cat is predisposed to fHCM. The true prevalence within the breed is not known, however it may be as high as 9.5-26.3\% [9]. Similar to human HCM, fHCM in MC cats exhibits incomplete penetrance and variable expressivity; thus, it is possible to find phenotypically normal mutations carriers $[10,11]$.

\section{C) Biomed Central}


Diagnosis of fHCM in MC cats should ideally be based on a positive family history, and a thorough echocardiographic assessment of several imaging planes of the heart with follow-ups. Genetic testing is currently of limited utility, as the clinical significance of being a mutation carrier has not been completely established. $\mathrm{fHCM}$ in $\mathrm{MC}$ cats is an excellent spontaneous animal model for human HCM, as the characteristics of the disease mimic the ones seen in human patients including the increased risk of sudden death $[7,12]$.

Mutations in the MYBPC3 gene, encoding the sarcomere cardiac protein Myosin Binding Protein $C$ (cMyBP-C), are associated with HCM in human and $\mathrm{fHCM}$ in MC and Ragdoll cats [4,5,13]. More than 240 $\mathrm{HCM}$-causing mutations in the $\mathrm{CMyBP}-\mathrm{C}$ protein have been reported from studies of human HCM [14], and cMyBP-C mutations are responsible for $\sim 30 \%$ of all human HCM cases [3,13,15].

Meurs et al. [4] identified a disease-causing missense mutation (c.91G > C) in the feline MYBPC3 gene in a colony of $\mathrm{MC}$ cats with $\mathrm{fHCM}$. The mutation causes the substitution of an alanine for a proline at residue 31 (p. A31P) of the cMyBP-C protein. Affected cats exhibit a broad phenotypic variation from mild to severe fHCM. Some cats have died before four years of age, where others were still alive at 8-12 years of age [4]. The frequency of this mutation has later been reported to be $34 \%$ among MC cats [16].

The objective of this study was to investigate the relationship between $\mathrm{fHCM}$ and $\mathrm{MC}$ cats heterozygous and homozygous for the p.A31P cMyBP-C mutation in a large cohort of $\mathrm{MC}$ cats. This study may contribute recommendations to $\mathrm{MC}$ breeding programs concerning the control of $\mathrm{fHCM}$.

\section{Methods}

\section{Clinical examinations}

A cohort of $332 \mathrm{MC}$ cats was prospectively included in the study at the Department of Small Animal Clinical Sciences, University of Copenhagen, Denmark. The cohort consisted of MC cats from MC breeders and owners who gave informed consent to participate. The study was approved by the ethics committee of the department. All cats were examined by 2-D and Mmode echocardiography in right lateral recumbency and imaged from below by one trained observer using a Vivid 7 Dimension ultrasonographic system equipped with a $10 \mathrm{~S}$ phased array transducer (4-11.5 MHz; GE Healthcare, Horten, Norway). Measurements of the left ventricle were obtained from M-mode imaging in standard echocardiographic right parasternal long axis fourchamber and short axis views at level of the chordae tendineae and according to the recommendations of the Echocardiography Committee of the Specialty of
Cardiology, American College of Veterinary Internal Medicine and the American Society of Echocardiography, respectively $[17,18]$. The $\mathrm{M}$-mode values of left ventricular dimensions were confirmed by measurements of multiple left ventricular wall segments from several 2-D views and cats were classified to have $\mathrm{fHCM}$ if the maximum diastolic wall thickness in any segment exceeded $5.5 \mathrm{~mm}$ in $>50 \%$ of segment length. Presence of an enlarged left atrium, systolic anterior motion of the septal leaflet of the mitral valve, left ventricular endsystolic cavity obliteration and enlarged papillary muscles further strengthened the diagnosis of fHCM. Cats were considered to be $\mathrm{fHCM}$ negative if the diastolic 'left ventricular free wall' (LVFW) and diastolic 'interventricular septum' (IVS) measured $<5 \mathrm{~mm}$ and no other cardiac abnormalities could be found. Cats were categorized as equivocal if they had a normal wall thickness $(<5.5 \mathrm{~mm})$ and displayed papillary muscle hypertrophy.

An fHCM screening form, from PawPeds international health programme [19], was filled out immediately after the examination and all images were stored digitally for later off-line analysis. All values represented the average of three consecutive beats. The method used to measure left atrium and aorta has previously been described in dogs [20].

The p.A31P cMyBP-C genotype of all cats was unknown to the observer at the echocardiographic examination and later offline analysis.

\section{Laboratory studies}

DNA was extracted from ethylenediaminetetraacetic acid stabilized blood or full blood using automated DNA purification by MAXWELL ${ }^{\circledR}$ (Promega, Nacka, Sweden) according to manufacturer's instructions. Where blood was not available (3\% of all samples), DNA was obtained and extracted using a MasterAmp ${ }^{\mathrm{TM}}$ Buccal Swab Kit (VWR \& Bie \& Berntsen, Herlev, Denmark) after the manufacturer's instructions. The feline $M Y B P C 3$ gene sequence was obtained from Ensembl (ENSFCAG00000002530) [21]. Amplification of genomic DNA was performed using the following primer set: exonic forward primer 5'-agccttcagcaagaagcca-3' and exonic reverse primer 5'-caaacttgaccttggaggagc-3'. The polymerase chain reaction (PCR) was carried out with 1 $\mu \mathrm{l}$ genomic DNA $(\sim 50 \mathrm{ng} / \mu \mathrm{l})$ in a volume of $25 \mu \mathrm{l}$ containing $1 \mu \mathrm{l} 20 \mathrm{pmol} / \mu \mathrm{l}$ primer mix (DNA technology AS, Aarhus, Denmark) $2.5 \mu \mathrm{l} 10 \times$ PCR buffer $(15 \mathrm{mM}$ $\mathrm{MgCl}_{2}$ ) (Qiagen, Copenhagen, Denmark), $0.5 \mu \mathrm{dNTP}$ mix $10 \mathrm{mM}$ solution (GE Healthcare Life Sciences, Brondby, Denmark), $0.2 \mu$ l Hot star polymerase (Qiagen, Copenhagen, Denmark) and $5 \mu \mathrm{l}$ Q-buffer (Qiagen, Copenhagen, Denmark). Samples were heat activated at $95^{\circ} \mathrm{C}$ for $15 \mathrm{~min}$ followed by 35 cycles: $95^{\circ} \mathrm{C}$ for $30 \mathrm{sec}$, 
$58^{\circ} \mathrm{C}$ for $30 \mathrm{sec}, 72^{\circ} \mathrm{C}$ for $1 \mathrm{~min}$ and a final step of elongation $\left(72^{\circ} \mathrm{C}\right.$ for $\left.7 \mathrm{~min}\right)$. PCR products were visually verified and thereafter treated with Exonuclease I (Medinova Scientific, Glostrup, Denmark). Mutation screening was carried out by direct DNA sequencing using BigDye ${ }^{\circledR}$ technology (GE Healthcare Life Sciences, Brondby, Denmark) on an ABI 3730 sequencer (Applied Biosystems, Naerum, Denmark). PCR products were sequence in both directions using the respective forward and reverse primers.

\section{Statistics}

The Chi-square test $\left(\chi^{2}\right)$ was used to evaluate if the genotype distribution was in Hardy-Weinberg equilibrium, a $P$-value $<0.05$ indicated significance. The clinical significance of the p.A31P cMyBP-C protein mutation was determined by looking at the probability of developing fHCM when comparing the heterozygous and the homozygous mutation carriers with the wild type cats using odds ratio calculation and the $95 \%$ confidence interval (95\% Cfi) was established.

When data were plotted, the measurements of age, weight, diastolic IVS and LVFW, systolic LVFW and the ratio of left atria over aorta (LA/Ao) all followed a nonnormal distribution, thus Kruskal-Wallis test was used to compare the medians of the data. A $P$-value $<0.05$ was considered statistically significant.

Spearman correlation was used to test if diastolic IVS and LVFW correlated with age for cats being homozygous for the mutation (Figure 1 ), a $P$-value $<0.05$ was considered significant.

\section{Results}

\section{The Maine Coon cohort}

The MC cohort consisted of 332 cats, 118 males with a median age of 1.5 years of age $(95 \%$ range $0.7 ; 6.0)$ and a median weight of $6.0 \mathrm{~kg}$ (95\% range $4.0 ; 8.8)$ and 214 queens with a median age of 2.0 years (95\% range 1.0; 5.5 ) and a median weight of $4.4 \mathrm{~kg}$ (95\% range $3.4 ; 6.0$ ). Presentation of the cohort can be seen in Tables 1 and 2 , where the cats were categorized according to their fHCM clinical presentation and p.A31P cMyBP-C, respectively.

The cats were categorized into three groups: $\mathrm{fHCM}$ positive, fHCM negative and equivocal cats. The fHCM positive group consisted of 21 cats (14 males). The equivocal group consisted of 26 cats (14 males) and the remaining 285 cats were classified as fHCM negative (90 males). No significant differences in age was found between the three groups $(P>0.05)$. The weight of fHCM positive cats was significantly higher than $\mathrm{HCM}$ negative cats $(P<0.05)$, no difference in weight was found between the $\mathrm{fHCM}$ cats and cats with equivocal status $(P>0.05)$.

\section{Characterisation of the fHCM positive MC cats}

Twenty-one cats were diagnosed with $\mathrm{fHCM}$, making the prevalence $6.3 \%$ - and $14.2 \%$ when the equivocal group was added to the fHCM affected group. However, for the male MC cats alone the fHCM prevalence was $11.9 \%$ and a further $11.9 \%$ were categorized as equivocal (a total of $23.7 \%$ ). See Table 3 for genotype and phenotype distribution.

In total, $13 \mathrm{fHCM}$ positive cats $(62 \%)$ had a cardiac murmur (3 with grade I, 4 with grade II, 5 with grade III, 1 with grade IV). Systolic anterior movement (SAM) of the mitral valve was observed in $52 \%$ of $\mathrm{fHCM}$ positive cats and end-systolic cavity obliteration was observed in $43 \%$ of fHCM positive cats. The diagnostic findings for the three groups are summarized in Table 1 . The wall thickness and the inner diameter of the ventricle given in Tables 1, 2 and 4 reflect standard measurement with M-mode echocardiography from a right parasternal short axis view at level of the chordae tendinae. Localized and asymmetrical thickening of the myocardium can be missed with a standard M-mode projection in all cats, thus they were also measured by 2-D echocardiography. The echocardiographic measurements and M-mode echocardiograms of hearts from an fHCM positive and a negative $\mathrm{MC}$, respectively, are shown in Figures 2 and 3.

The equivocal cats were mainly characterized by normal wall thickness $(<5.5 \mathrm{~mm})$ and papillary muscle size, with or without end-systolic cavity obliteration. In odds ratio calculations the equivocal cats were first added to the group of $\mathrm{fHCM}$ positive cats and afterward added to the group of $\mathrm{fHCM}$ negative cats for the same calculations.

\section{p.A31P cMyBP-C genotyping}

All cats were genotyped with respect to the cMyBP-C mutation (Table 3). Eighteen MC cats were homozygous, $89 \mathrm{MC}$ cats were heterozygous and $225 \mathrm{MC}$ cats were wild type (no mutation). 10 out of $21 \mathrm{MC}$ cats with an $\mathrm{fHCM}$ diagnosis did not carry the mutation. The histogram in Figure 4A shows the fraction of $\mathrm{fHCM}$ and equivocal cases in the three different genotypes.

The mutated MYBPC3 gene-allele (c.91C) was found to be the minor allele with an allele frequency of 0.19 . The genotype distribution was not in Hardy-Weinberg equilibrium $\left(P<0.05, \chi^{2}\right.$ test $)$.

\section{The significance of the p.A31P cMyBP-C mutation}

Odds ratio calculations were used to determine the probability of developing $\mathrm{fHCM}$ in all cats with the mutation. The odds ratio was calculated for heterozygous and homozygous carriers of p.A31P cMyBP-C between $\mathrm{fHCM}$ affected and non-affected cats, using 


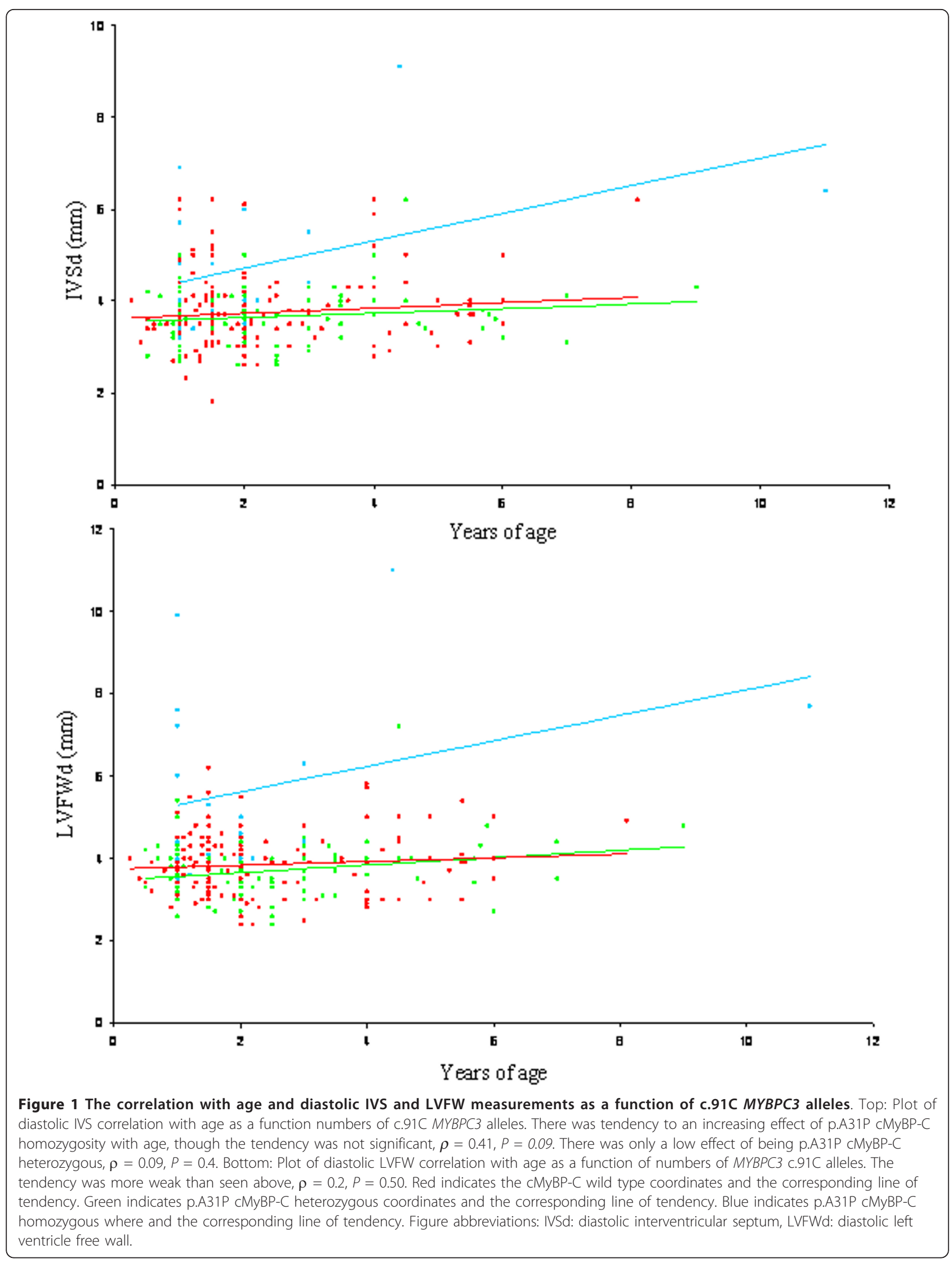


Table 1 Presentation of Main Coon cat cohort

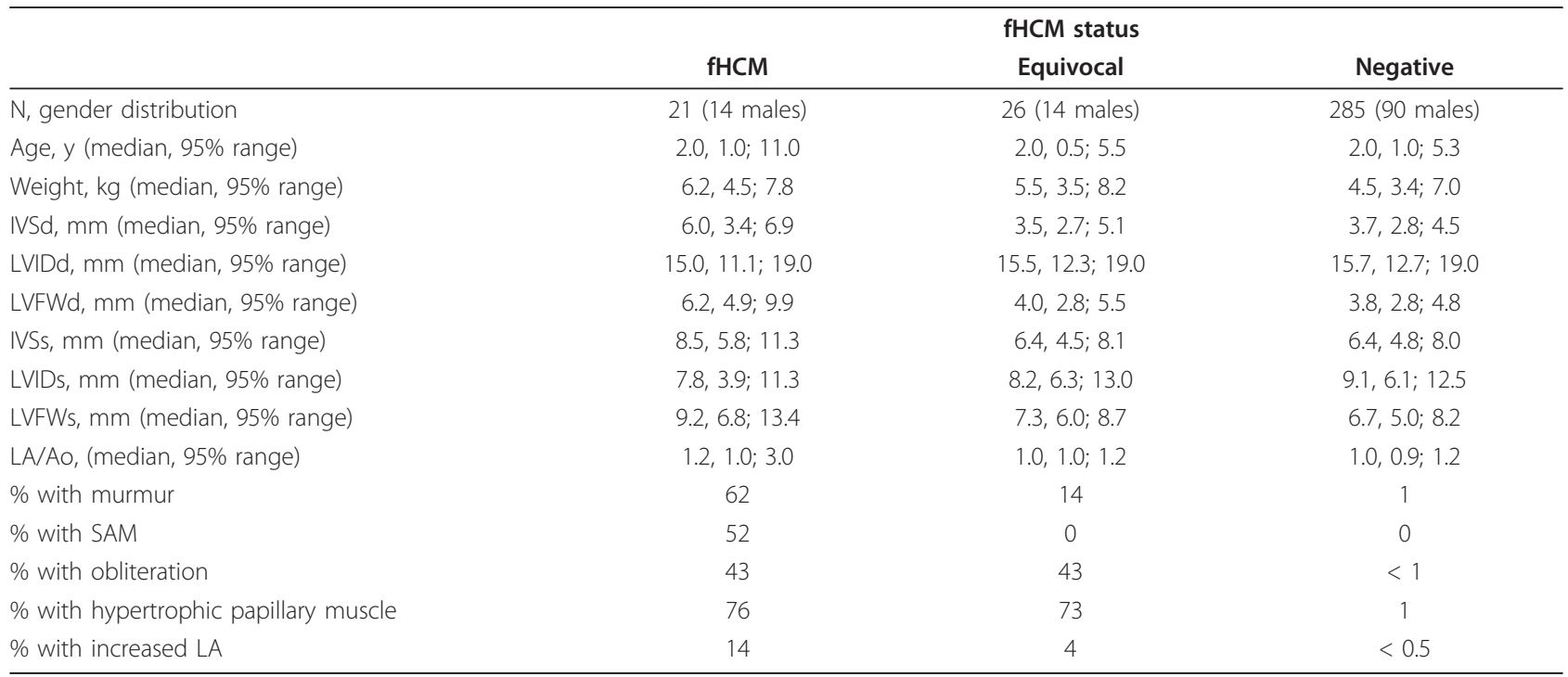

IVSd: diastolic intraventricular septum, LVIDd: diastolic left ventricular inner diameter, LVFWd: diastolic left ventricular free wall, IVSs: systolic intraventricular septum, LVIDs: systolic left ventricular inner diameter, LVFWs: systolic left ventricular free wall, LA/Ao: left atrium over aorta.

mutation wild type cats as a control group. The odds ratio of having fHCM (or being equivocal) for all cats carrying the C allele (c.91C) was found to be 1.9 (95\% Cfi: $1.0 ; 3.6)$. The odds ratios of having fHCM for homozygous and heterozygous mutation carriers were 12.1 (95\% Cfi: 4.3; 33.9) and 1.0 (95\% Cfi: 0.5; 2.1), respectively. The same odds ratio calculations, with the equivocal cats added to the group of $\mathrm{FHCM}$ negative cats revealed an odds ratio for cats carrying the $\mathrm{C}$ allele (c.91C) to be 2.5 (95\% Cfi: 1.0; 6.0). For homozygous and heterozygous cats the odds ratios were found to be
21.6 (95\% Cfi: $7.0 ; 66.2)$ and 0.5 (95\% Cfi: $0.1 ; 2.3)$, respectively.

The fHCM prevalence for the disease was found to be $2.4 \%$ in heterozygous cats and $9.1 \%$ of heterozygous cats were classified as equivocal. Among p.A31P cMyBP-C homozygous $\mathrm{MC}$ cats, $50 \%$ had $\mathrm{fHCM}$ and a further $11 \%$ were categorized as equivocal (Table 3). Table 5 gives an overview of the fHCM penetrance and the fHCM odds ratio of the group of heterozygous cats, the homozygous cats and both combined. The $\mathrm{fHCM}$ odds ratios of all three categories were compared to cMyBP-C wild type cats.

Table 2 Presentation of the Main Coon cat cohort categorised in groups depending on the A31P CMYBP-C genotype

\begin{tabular}{|c|c|c|c|c|c|}
\hline & \multicolumn{3}{|c|}{ cMyBP-C p.A31P genotype } & \multicolumn{2}{|c|}{$P$-value mutation negative vs } \\
\hline & Homozygous (C/C) & Heterozygous (G/C) & Wild type (G/G) & Homozygous & Heterozygous \\
\hline n, gender distribution & 18 (12 males) & 89 (25 males) & 225 (81 males) & - & - \\
\hline Age, y (median, 95\% range) & $1.5,1.0 ; 11.0$ & $2.0,0.9 ; 5.9$ & $1.7,1.0 ; 5.0$ & 0.48 & 0.14 \\
\hline Weight, kg (median, 95\% range) & $5.9,3.4 ; 7.0$ & $4.6,3.5 ; 7.8$ & $4.5,3.4 ; 7.2$ & 0.04 & 0.32 \\
\hline IVSd, mm (median, 95\% range) & $4.2,3.2 ; 9.1$ & $3.7,2.8 ; 5.5$ & $3.7,2.8 ; 5.1$ & 0.002 & 0.70 \\
\hline LVIDd, mm (median, 95\% range) & $15.6,10.2 ; 18.4$ & $15.9,12.8 ; 19.0$ & $15.6,12,3 ; 19.0$ & 0.88 & 0.59 \\
\hline LVFWd, mm (median, 95\% range) & $5.1,3.6 ; 11.0$ & $3.7,2.7 ; 4.8$ & $4.0,2.9 ; 5.0$ & $<0.001$ & 0.10 \\
\hline IVSs, mm (median, 95\% range) & $7.8,5.0 ; 11.3$ & $6.3,4.8 ; 8.1$ & $6.4,4.8 ; 8.4$ & 0.04 & 0.29 \\
\hline LVIDs, mm (median, 95\% range) & $8.8,4.0 ; 13.0$ & $9.0,5.7 ; 12.6$ & $9.0,6.1 ; 12.4$ & 0.23 & 0.80 \\
\hline LVFWs, mm (median, 95\% range) & $8.9,5.5 ; 13.4$ & $6.7,5.1 ; 8.3$ & $6.7,5.1 ; 8.7$ & 0.016 & 0.90 \\
\hline LA/Ao, (median, 95\% range) & $1.1,0.9 ; 4.0$ & $1.0,0.9 ; 1.1$ & $1,0.9 ; 1.2$ & 0.0004 & 0.31 \\
\hline$\%$ with murmur & 50 & 2 & 4 & & \\
\hline$\%$ with SAM & 44 & 1 & 0 & & \\
\hline$\%$ with obliteration & 44 & 4 & 5 & & \\
\hline$\%$ with hypertrophic papillary muscle & 56 & 9 & 8 & & \\
\hline$\%$ with increased LA & 22 & 0 & $<1$ & & \\
\hline
\end{tabular}

IVSd: diastolic intraventricular septum, LVIDd: diastolic left ventricular inner diameter, LVFWd: diastolic left ventricular free wall, IVSs: systolic intraventricular septum, LVIDs: systolic left ventricular inner diameter, LVFWs: systolic left ventricular free wall, LA/Ao: left atrium over aorta. 
Table 3 Distribution of the fHCM negative, the equivocal and the fHCM positive cats in respect to $\mathrm{p} . \mathrm{A} 31 \mathrm{P}$ genotype

\begin{tabular}{lccc}
\hline & Negative & Equivocal & fHCM \\
\hline Wild type (G/G) & 200 & 16 & 10 \\
Heterozygous (G/C) & 78 & 8 & 2 \\
Homozygous (C/C) & 7 & 2 & 9 \\
\hline
\end{tabular}

The fHCM diagnostic parameters compared between groups of the three different genotypes are summarized in Table 2 .

Figure 1 represents plots of the echocardiographic measurements of diastolic IVS and LVFW as a function of age and p.A31P cMyBP-C heterozygous or homozygous status. There was no significant correlation between age and diastolic IVS or LVFW in cats heterozygous for the mutation or in wild type cats. There was a tendency for diastolic IVS $(\rho=0.4, P=0.09$ ) to increase with age for cats homozygous for the mutation.

A small cohort consisting of all cats over 4 years of age (with a median age of 4.9 years, $95 \%$ range $4 ; 8.1$ ) was used to examine the significance of being affected by the p.A31P cMyBP-C mutation in middle-aged cats. The small cohort consisted of 50 cats; six of which had been positively diagnosed with fHCM. Within this cohort, we identified 14 cats being heterozygous and two cats being homozygous for the mutation. One equivocal cat and one fHCM positive cat were among the heterozygous cats and both homozygous cats were fHCM positive. The odds ratio of being fHCM positive for heterozygous mutation carriers over four years of age was found to be 0.64 (95\% Cfi 0.1-3.6) - when the equivocal cats were categorized as $\mathrm{fHCM}$ affected. Categorizing the equivocal cats as non-affected resulted in an odds ratio of 0.8 (95\%Cfi: 0.1-8.4). The relative distribution of $\mathrm{fHCM}$ and equivocal cats as a function of the p.A31P cMyBP-C genotype is shown in Figure 4B. Table 4 presents the echocardiographic measurements corresponding to either heterozygous or non-mutation carriers.

\section{Discussion}

We investigated the clinical significance of being heterozygous or homozygous for the p.A31P cMyBP-C mutation in a cohort of young MC cats. We did not find a significant odds ratio for the development of fHCM for cats heterozygous for the mutation. However, we did find a significantly high odds ratio for cats homozygous for the mutation and so a high probability for developing fHCM. Based on these results the penetrance in heterozygous cats must be considered very low in young cats and high in young cats homozygous for the mutation.

Additionally, we found that diastolic IVS thickness had a tendency to progress with age in p.A31P cMyBP-C homozygous cats compared to heterozygous and wild type cats. A reduced number of homozygous cats due to fHCM related death might explain why the correlation between increasing age and increasing diastolic IVS or LVFW was not significant.

To compensate for the young mean age of the cohort we investigated a smaller group consisting of 50 cats, all over four years of age with a median of 4.9 years of age (95\% range: $4 ; 8.1$ ). In this small cohort, 14 cats were heterozygous and a further two were homozygous.

Table 4 Echocardiographic characteristics for cats over 4 years of age

\begin{tabular}{|c|c|c|c|}
\hline & \multicolumn{2}{|c|}{ cMyBP-C p.A31P genotype } & \multirow[t]{2}{*}{$P$-value } \\
\hline & Heterozygous (G/C) & Wild type (G/G) & \\
\hline$n$, gender distribution & 14 (4 males) & 34 (14 males) & - \\
\hline Age, y (median, 95\% range) & $5.7,4.0 ; 9.0$ & $4.5,4.0 ; 6.0$ & 0.17 \\
\hline Weight, kg (median, 95\% range) & $6.0,4.5 ; 8.8$ & $5.1,8.7 ; 3.5$ & $>0.05$ \\
\hline IVSd, mm (median, 95\% range) & $3.7,3.1 ; 6.2$ & $3.9,2.9 ; 6.2$ & 0.40 \\
\hline LVIDd, mm (median, 95\% range) & $16.6,13.8 ; 19.3$ & $15.9,13.8 ; 1.9$ & 0.20 \\
\hline LVFWd, mm (median, 95\% range) & $4.0,2.7 ; 7.2$ & $4.0,2.9 ; 5.8$ & 0.70 \\
\hline IVSs, mm (median, 95\% range) & $6.6,5.0 ; 12.4$ & $6.7,5.0 ; 9.0$ & 0.82 \\
\hline LVIDs, mm (median, 95\% range) & $9.6,3.9 ; 12.9$ & $10.0,6.3 ; 14.0$ & 0.83 \\
\hline LVFWs, mm (median, 95\% range) & $7.0,5.2 ; 9.2$ & $7.0,4.1 ; 9.8$ & 0.08 \\
\hline LA/Ao, (median, 95\% range) & $1.0,0.9 ; 1.3$ & $1.0,0.9 ; 1.4$ & 0.06 \\
\hline$\%$ with murmur & 7 & 0 & \\
\hline$\%$ with SAM & 7 & 0 & \\
\hline$\%$ with obliteration & 14 & 3 & \\
\hline$\%$ with hypertrophic papillary muscle & 7 & 12 & \\
\hline$\%$ with increased LA & 0 & 0 & \\
\hline
\end{tabular}

IVSd: diastolic intraventricular septum, LVIDd: diastolic left ventricular inner diameter, LVFWd: diastolic left ventricular free wall, IVSs: systolic intraventricular septum, LVIDs: systolic left ventricular inner diameter, LVFWs: systolic left ventricular free wall, LA/Ao: left atrium over aorta. 

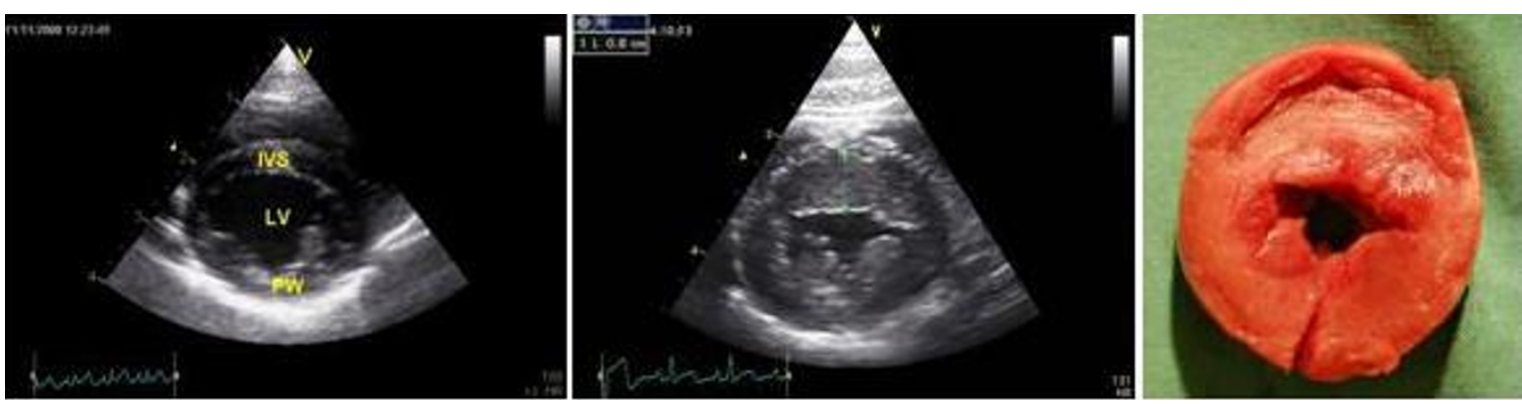

Figure 2 2-D echocardiographic recordings of papillary muscles from a normal and an fHCM affected cat. Left illustration: Right parasternal short axis of the left ventricle with papillary muscles view from a normal MC cat. Middle illustration: Right parasternal short axis of the left ventricle with papillary muscles view from a cat with severe hypertrophic cardiomyopathy. Right illustration: Gross heart specimen from a cat with severe concentric hypertrophy due to fHCM. Figure abbreviations: IVS: Intraventricular septum, LV: left ventricle and PW: posterior wall (free wall)

Again, no significant association between p.A31P cMyBP-C and $\mathrm{fHCM}$ was found in heterozygous cats. This again indicated that p.A31P cMyBP-C associated $\mathrm{fHCM}$ was a disease with a very low penetrance also in middle-aged cats heterozygous for the mutation and highly penetrant for cats homozygous for the mutation. We propose that the p.A31P cMyBP-C mutation results in an inheritance patterns that resembles a recessive form of inheritance in young MC cats.

However, the clinical significance of the heterozygous mutation carrier status must be established in a cohort of older cats before conclusions regarding mode of inheritance are finalized and firm breeding recommendations made. Therefore, follow-up studies of the cohort are recommended with a delay of 2-5 years from this study to evaluate the clinical course of the heterozygous in respect to the probability of late-onset fHCM. Very recently, Wess et al. [11] stated that the p.A31P mutation is less pathogenic than reported so far based upon a cohort of $82 \mathrm{MC}$ cats (mean age approx. 70-72 months). However, we find the number of homozygous cats in that study were too low $(\mathrm{n}=3)$ to reliably assess the fHCM risk associated with this genotype [11]. The low number of homozygous cats might be the result of fHCM-associated yearly death. That study supports our results concerning heterozygous mutation carriers and fHCM development. However, another study has reported tissue Doppler imaging evidence of diastolic dysfunction in MC cats heterozygous for the mutation indicating that even though there may not be overt evidence of $\mathrm{fHCM}$ there is evidence of occult $\mathrm{fHCM}$ in these cats [10]. If occult disease is taken into consideration then an autosomal dominant mode of inheritance needs to be considered.
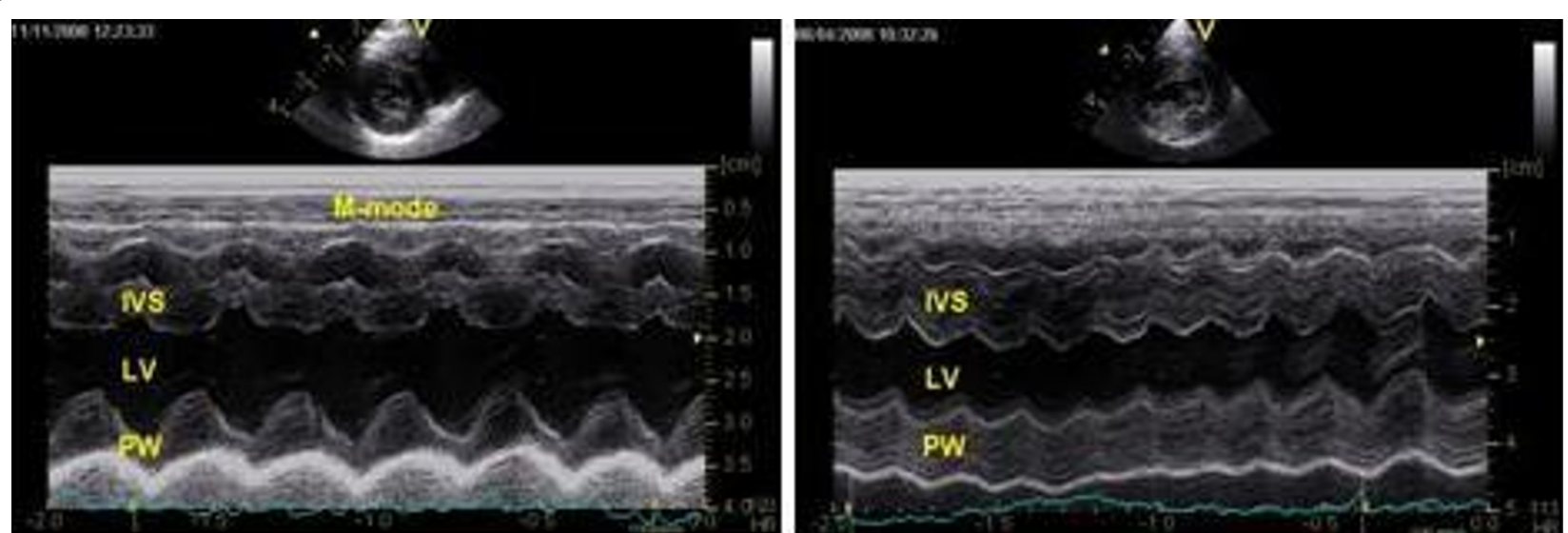

Figure 3 Illustration of M-mode echocardiographic recordings from a normal and an fHCM affected cat. Left illustration: M-mode echocardiogram recorded from an MC cat at the level of chordae tendinae shows normal left ventricular internal dimension, septum and posterior wall of the left ventricle. Right: M-mode echocardiogram from and an MC cat with severe fHCM with atrial fibrillation (HR 325/min), congestive heart failure and tromboembolic disease, recorded the level of the chordae tendinae to assess left ventricular dimensions shows severe thickening of both the septum and posterior wall of the left ventricle. Figure abbreviations: IVS: Intraventricular septum, LV: left ventricle and PW: posterior wall. 


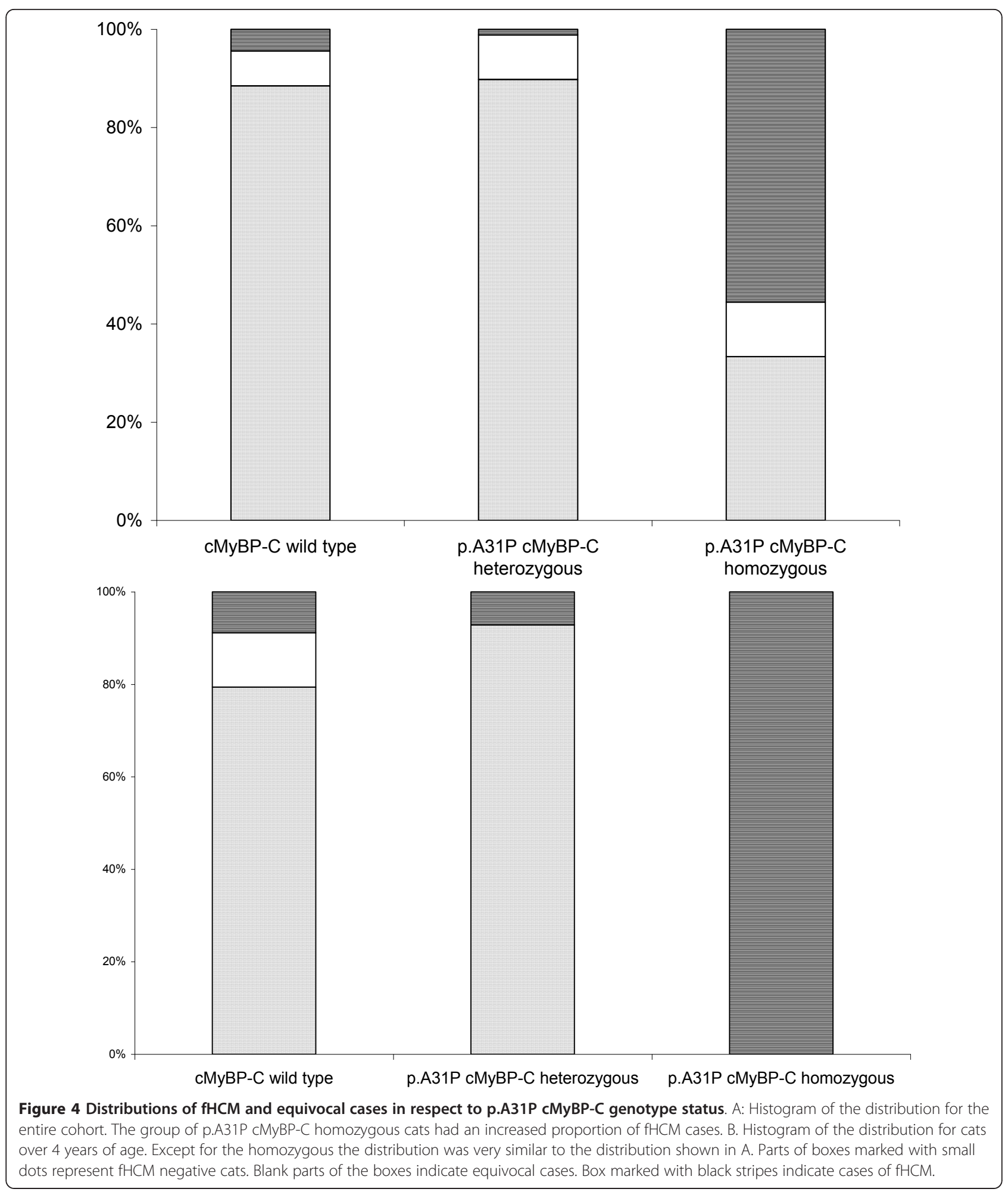

Recessive, or gene dosage dependent, mode of inheritance has previously been shown to be the case in some human patients with cMyBP-C associated HCM [22]. It can not be excluded that the p.A31P cMyBP-C mutation may exhibit a dominant pattern of inheritance pattern in a single colony of cats as previously described [4]. This may be a consequence of various factors, which may modify the phenotype. It is possible that those pedigrees with cats heterozygous for the p.A31P mutation and affected with $\mathrm{fHCM}$ may represent digenic inheritance 
Table 5 The fHCM odds ratio and fHCM prevalence of the three genotype categories: heterozygous, homozygous and the hetero- and homozygous combined

\begin{tabular}{|c|c|c|c|c|}
\hline Genotype & fHCM prevalence & fHCM + equivocal prevalence & fHCM Odds ratio* & fHCM + equivocal odds ratio \\
\hline All $(G / G)+(G / C)+(C / C)$ & $6.3 \%$ & $14.2 \%$ & - & - \\
\hline Heterozygous (G/C) & $2.3 \%$ & $12.8 \%$ & 0.5 (95\% Cfi: $0.1 ; 2.3)$ & 0.8(95\% Cfi: $0.3 ; 1.8)$ \\
\hline Homozygous (C/C) & $50 \%$ & $61.1 \%$ & 21.6 (95\% Cfi: 7.0; 66.2) & 12.1 (95\% Cfi: 4.3; 33.9) \\
\hline All carriers $(\mathrm{G} / \mathrm{C})+(\mathrm{C} / \mathrm{C})$ & $10.3 \%$ & $19.8 \%$ & 2.5 (95\% Cfi: 1.0; 6.0) & 2.9 (95\% Cfi: 1.6; 5.5) \\
\hline
\end{tabular}

*The equivocal cats have been added to the non-affected cats.

In all three categories the odds ratio was calculated as compared to the group of wild type cats.

or compound heterozygosity where another yet undiscovered mutation is present with the p.A31P cMyBP-C mutation. Digenic or compound heterozygosity is present in $8-30 \%$ of human HCM cases [13,15,23]. This suggests the need to conduct a mutation screening of all the HCM associated sarcomeric genes in MC cats.

Seven out of $21 \mathrm{MC}$ cats with an fHCM diagnosis were not carrying the mutation, revealing that the etiology of fHCM is heterogeneous (i.e. at least one more cause is present) in the MC breed. Human HCM is associated with one or more mutations in at least 11 cardiac sarcomere genes [15]. In total, eight cardiac sarcomere genes associated with human HCM have been screened for fHCM disease-causing mutations in 14 cats from five different breeds [24]. The study did not identify any new disease-causing mutations in the sarcomere genes MYH7, MYBPC3, TNNT2, TNNI3, TPM1, MYL2, $M Y L 3$ and $A C T C$. However, these genes should still be considered as candidate genes for $\mathrm{fHCM}$, as the cohort used in the study is very small [24]. By analogy to human HCM, it would seem very unlikely to identify mutations in these genes in a small patient group as the genes are - individually - rarely affected $[13,15]$.

Defining a "normal heart" is a complicated clinical task that involves serial measurements and collecting a sufficient variety of pre-clinical and clinical information. Each specific heart disease, in principle, needs a specific reference interval or decision limits (cut-off points). Decision limits for fHCM with diastolic LVFW and IVS $>6 \mathrm{~mm}$ may impose difficulties in screening for fHCM in phenotypically normal "carriers" or in cats with mutations that cause late-onset fHCM. In early stages of disease, a high percentage of false negative results may be the consequence of too high a cut-off point.

The group of equivocal MC cats may contain a high number of both "false positive" and "false negative" cats. The category contains cats with normal wall thickness $(<5.5 \mathrm{~mm})$ and papillary muscle hypertrophy, with or without obliteration. Consequently, the term "equivocal" was given to cats in which the heart showed some of the echocardiographic signs suggestive of fHCM, but without being distinctive enough to classify the cat as fHCM positive. These cats were in a grey zone and follow-up echocardiographic examination was always recommended to this group.

The present reference interval was established in a homogeneous MC population at large. No "normal" female or male cats had wall thickness $>5 \mathrm{~mm}$ and only three out of twenty-six equivocal cats were in the range from 5-5.5 $\mathrm{mm}$. Although a lowering of a decision limit from 6 to $5.5 \mathrm{~mm}$ may cause slightly higher false positive results, we found that a $5.5 \mathrm{~mm}$ upper limit was a more appropriate value for screening for fHCM. This was a finding in agreement with a previous study where $5.0 \mathrm{~mm}$ is suggested as normal upper limit for myocardial thickness in $\mathrm{MC}$ [9].

The p.A31P cMyBP-C genotype distribution was not in Hardy-Weinberg equilibrium, indicating that there was a bias of selection in the MC cohort. In addition, the disequilibrium could possibly be explained by a reduced number of homozygous cats, which supports that the $\mathrm{p}$. A31P cMyBP-C mutation was disease-causing and resulted in an increased mortality even in young cats. This is comparable with the findings in human HCM, where an early clinical debut of HCM is associated with a very poor prognosis [25]. Breeders volunteered their cats to be enrolled in the study, thus breeders with no interest in $\mathrm{fHCM}$ and $\mathrm{fHCM}$ genetics were not likely to participate. Furthermore, MC cats from breeding programs are bred based on certain selection criteria. Breeders with a commercial interest only use the strongest male and female cats for reproduction. That the prevalence of fHCM and of the p.A31P cMyBP-C mutation found in our cohort is similar to previously reported prevalence reduces bias though $[9,16,26]$.Finally, only one-third of the cats in our cohort were males, this bias may result in underestimation of the $\mathrm{fHCM}$ prevalence, as $\mathrm{fHCM}$ is more common in male cats.

\section{Conclusions}

In conclusion, p.A31P cMyBP-C associated $\mathrm{fHCM}$ is a disease with very low penetrance in young heterozygous cats. Our results support the pathogenic role of p.A31P when two affected gene alleles are present in a MC cat. Homozygosity of the $\mathrm{cMyBP}-\mathrm{C}$ mutation only explained $43 \%$ of $\mathrm{fHCM}$ cases in the MC cohort therefore we 
recommend further large-scale genetic studies to identify potential disease-causing mutations in genes including the sarcomere genes most commonly involved in human HCM. Furthermore, due to the high probability of developing fHCM in the p.A31P cMyBP-C homozygous cats the 'production' of homozygous $\mathrm{MC}$ cats should be avoided. Thus, although genotyping of the $\mathrm{p}$. A31P cMyBP-C mutation can not stand alone in limiting $\mathrm{fHCM}$ in $\mathrm{MC}$, it is very important that breeders are aware of the genotype status and breeders should be informed of breeding recommendations. Breeding recommendations concerning this genetic variant are still controversial.

\section{List of abbreviations}

c.91G > C: guanine substituted for a cytosine at the $91^{\text {th }}$ nucleotide of the coding gene sequence; Cfi: confidence interval; CMyBP-C: cardiac myosin binding protein C; fHCM: feline HCM; HCM: Hypertrophic cardiomyopathy; IVS: interventricular septum; LA/Ao: ratio of left atria diameter over aorta; LV: left ventricle; LVFW: left ventricular free wall; LVID: left ventricular inner diameter; MC: Maine Coon; p.A31P: alanine substituted for a proline at residue 31; PW: posterior wall; OR: Odds ratio; SAM: systolic anterior movement; Range: 95\% interquartile range.

\section{Acknowledgements}

This work was supported by the Novo Nordisk Foundation. Picture of the heart from an affected MC cat was kindly provided by Jakob L Willesen. Veterinary nurse Michelle J Dupont is acknowledged for her help in getting blood samples from our feline patients. Cand. scient. Paula Hedley is highly acknowledged for her assistance in the final proof-reading phase and Severin Olesen for his help with statistic. All participating MC owners and breeders are acknowledged for their participation in the study.

\section{Author details}

'Department of Clinical Biochemistry and Immunology, Statens Serum Institut, Artillerivej 5, DK-2300 Copenhagen, Denmark. ²Department of Small Animals Clinical Sciences, Faculty of Life Science, University of Copenhagen Dyrlægevej 46, DK-1870 Frederiksberg C, Denmark.

\section{Authors' contributions}

MTNG has designed the study, performed the genetic study and genetic data analysis, performed the statistic data analysis of the clinical data and had the primary responsibility concerning drafting the manuscript. SG and JK both participated in sampling the clinical data and drafting the manuscript, JK also participated in study design. MC participated study design, genetic analysis and drafting the manuscript. All authors read and approved the final manuscript.

\section{Conflict of interest statement}

None of the authors of this paper has a financial or personal relationship with other people or organisations that could inappropriately influence or bias the content of the paper

Received: 19 April 2010 Accepted: 9 February 2011

Published: 9 February 2011

\section{References}

1. Alcalai R, Seidman JG, Seidman C: Genetic basis of hypertrophic cardiomyopathy: from bench to the clinics. J Cardiovasc Electrophysiol 2008, 19:104-110.

2. Keren A, Syrris P, McKenna WJ: Hypertrophic cardiomyopathy: the genetic determinants of clinical disease expression. Nat Clin Pract Cardiovasc Med 2008, 5:158-168.

3. Marian AJ: Genetic determinants of cardiac hypertrophy. Curr Opin Cardiol 2008, 23:199-205.
4. Meurs KM, Sanchez X, David RM, Bowles NE, Towbin JA, Reiser PJ, Kittleson JA, Munro MJ, Dryburgh K, MacDonald KA, Kittleson MD: A cardiac myosin binding protein $\mathrm{C}$ mutation in the Maine Coon cat with familial hypertrophic cardiomyopathy. Hum Mol Genet 2005, 14:3587-3593.

5. Meurs KM, Norgard MM, Ederer MM, Hendrix KP, Kittleson MD: A substitution mutation in the myosin binding protein $C$ gene in ragdoll hypertrophic cardiomyopathy. Genomics 2007, 90:261-264.

6. Richard P, Charron P, Carrier L, Ledeuil C, Cheav T, Pichereau C, Benaiche A, Isnard R, Dubourg O, Burban M, Gueffet JP, Millaire A, Desnos M, Schwartz K, Hainque B, Komajda M: Hypertrophic cardiomyopathy: distribution of disease genes, spectrum of mutations, and implications for a molecular diagnosis strategy. Circulation 2003, 107:2227-2232.

7. Kittleson MD, Meurs KM, Munro MJ, Kittleson JA, Liu SK, Pion PD, Towbin JA: Familial hypertrophic cardiomyopathy in maine coon cats: an animal model of human disease. Circulation 1999, 99:3172-3180.

8. Hyun C, Filippich LJ: Molecular genetics of sudden cardiac death in small animals - a review. Vet J 2006, 171:39-50.

9. Gundler S, Tidholm A, Haggstrom J: Prevalence of myocardial hypertrophy in a population of asymptomatic Swedish Maine coon cats. Acta Vet Scand 2008, 50:22

10. Carlos SC, Chetboul V, Mary J, Tissier R, Abitbol M, Serres F, Gouni V, Thomas A, Pouchelon JL: Prospective echocardiographic and tissue Doppler imaging screening of a population of Maine Coon cats tested for the A31P mutation in the myosin-binding protein $C$ gene: a specific analysis of the heterozygous status. J Vet Intern Med 2009, 23:91-99.

11. Wess G, Schinner C, Weber K, Kuchenhoff H, Hartmann K: Association of $\mathrm{A} 31 \mathrm{P}$ and A74T polymorphisms in the myosin binding protein C3 Gene and hypertrophic cardiomyopathy in Maine Coon and other breed cats. J Vet Intern Med 2010, 24:527-532.

12. Fox PR, Liu SK, Maron BJ: Echocardiographic assessment of spontaneously occurring feline hypertrophic cardiomyopathy. An animal model of human disease. Circulation 1995, 92:2645-2651.

13. Andersen PS, Havndrup O, Bundgaard H, Larsen LA, Vuust J, Pedersen AK, Kjeldsen K, Christiansen M: Genetic and phenotypic characterization of mutations in myosin-binding protein C (MYBPC3) in 81 families with familial hypertrophic cardiomyopathy: total or partial haploinsufficiency. Eur J Hum Genet 2004, 12:673-677.

14. Biobase Biological Databases, Humane Gene Mutation Database. [http:// www.biobase-international.com/index.php?id=hgmddatabase].

15. Andersen PS, Havndrup $O$, Hougs L, Sorensen KM, Jensen M, Larsen LA, Hedley P, Thomsen AR, Moolman-Smook J, Christiansen M, Bundgaard H: Diagnostic yield, interpretation, and clinical utility of mutation screening of sarcomere encoding genes in Danish hypertrophic cardiomyopathy patients and relatives. Hum Mutat 2009, 30:363-370.

16. Fries $\mathrm{R}$, Heaney AM, Meurs KM: Prevalence of the myosin-binding protein C mutation in Maine Coon cats. J Vet Intern Med 2008, 22:893-896.

17. Thomas WP, Gaber CE, Jacobs GJ, Kaplan PM, Lombard CW, Moise NS, Moses BL: Recommendations for standards in transthoracic twodimensional echocardiography in the dog and cat. Echocardiography Committee of the Specialty of Cardiology, American College of Veterinary Internal Medicine. J Vet Intern Med 1993, 7:247-252.

18. Lang RM, Bierig M, Devereux RB, Flachskampf FA, Foster E, Pellikka PA Picard MH, Roman MJ, Seward J, Shanewise JS, Solomon SD, Spencer KT, Sutton MS, Stewart WJ: Recommendations for chamber quantification: a report from the American Society of Echocardiography's Guidelines and Standards Committee and the Chamber Quantification Writing Group, developed in conjunction with the European Association of Echocardiography, a branch of the European Society of Cardiology. J Am Soc Echocardiogr 2005, 18:1440-1463.

19. Pawpeds. [http://pawpeds.com/healthprogrammes/hcmtest.html]

20. Haggstrom J, Kvart C, Hansson K: Heart sounds and murmurs: changes related to severity of chronic valvular disease in the Cavalier King Charles spaniel. J Vet Intern Med 1995, 9:75-85.

21. Ensembl. [http://www.ensembl.org/Felis_catus/Info/Index].

22. Nanni L, Pieroni M, Chimenti C, Simionati B, Zimbello R, Maseri A, Frustaci A, Lanfranchi G: Hypertrophic cardiomyopathy: two homozygous cases with "typical" hypertrophic cardiomyopathy and three new mutations in cases with progression to dilated cardiomyopathy. Biochem Biophys Res Commun 2003, 309:391-398.

23. Van Driest SL, Vasile VC, Ommen SR, Will ML, Tajik AJ, Gersh BJ, Ackerman MJ: Myosin binding protein $\mathrm{C}$ mutations and compound 
heterozygosity in hypertrophic cardiomyopathy. J Am Coll Cardiol 2004, 44:1903-1910.

24. Meurs KM, Norgard MM, Kuan M, Haggstrom J, Kittleson M: Analysis of 8 sarcomeric candidate genes for feline hypertrophic cardiomyopathy mutations in cats with hypertrophic cardiomyopathy. J Vet Intern Med 2009, 23:840-843.

25. Badertscher A, Bauersfeld U, Arbenz U, Baumgartner MR, Schinzel A Balmer C: Cardiomyopathy in newborns and infants: a broad spectrum of aetiologies and poor prognosis. Acta Paediatr 2008, 97:1523-1528.

26. Paige CF, Abbott JA, Elvinger F, Pyle RL: Prevalence of cardiomyopathy in apparently healthy cats. J Am Vet Med Assoc 2009, 234:1398-1403.

doi:10.1186/1751-0147-53-7

Cite this article as: Godiksen et al:: Hypertrophic cardiomyopathy in young Maine Coon cats caused by the p.A31P CMyBP-C mutation - the clinical significance of having the mutation. Acta Veterinaria Scandinavica $201153: 7$

Submit your next manuscript to BioMed Central and take full advantage of:

- Convenient online submission

- Thorough peer review

- No space constraints or color figure charges

- Immediate publication on acceptance

- Inclusion in PubMed, CAS, Scopus and Google Scholar

- Research which is freely available for redistribution

Submit your manuscript at www.biomedcentral.com/submit 